2003. Delayed (>3 weeks) postnatal corticosteroids for chronic lung disease in preterm infants. Cochrane Database Syst. Rev. CD001145.

10. Heine, V.M., and Rowitch, D.H. 2009. Hedgehog signaling has a protective effect in glucocorticoidinduced mouse neonatal brain injury through an $11 \beta$ HSD2-dependent mechanism. J. Clin. Invest. 119:267-277

11. Varjosalo, M., and Taipale, J. 2008. Hedgehog: functions and mechanisms. Genes Dev. 22:2454-2472.

12. Gulino, A., Di Marcotullio, L., Ferretti, E., De Smaele, E., and Screpanti, I. 2007. Hedgehog signaling pathway in neural development and disease. Psychoneuroendocrinology 32(Suppl 1):S52-S56.

13. Ruiz i Altaba, A. 2006. Hedgehog-Gli signaling in buman disease. Landes Bioscience. Georgetown, Texas, USA. 228 pp.

14. Holmes, M.C., and Seckl, J.R. 2006. The role of 11 beta-hydroxysteroid dehydrogenases in the brain. Mol. Cell. Endocrinol. 248:9-14.

15. Lewis, P.M., et al. 2001. Cholesterol modification of sonic hedgehog is required for long-range signaling activity and effective modulation of signaling by Ptc1. Cell. 105:599-612.
16. Corcoran, R.B., and Scott, M.P. 2006. Oxysterols stimulate Sonic hedgehog signal transduction and proliferation of medulloblastoma cells. Proc. Natl. Acad. Sci. U. S. A. 103:8408-8413.

17. Dwyer, J.R., et al. 2007. Oxysterols are novel activators of the hedgehog signaling pathway in pluripotent mesenchymal cells. J. Biol. Chem. 282:8959-8968.

18. Bijlsma, M.F., et al. 2006. Repression of smoothened by patched-dependent (pro-)vitamin D3 secretion. PLoS Biol. 4:e232.

19. Lee, K., et al. 2006. Molecular mechanisms involved in progesterone receptor regulation of uterine function. J. Steroid Biochem. Mol. Biol. 102:41-50

20. Katayama, S., et al. 2006. The expression of Hedgehog genes (Ihh, Dhh) and Hedgehog target genes (Ptc1, Gli1, Coup-TfII) is affected by estrogenic stimuli in the uterus of immature female rats. Toxicol. Appl. Pharmacol. 217:375-383.

21. Prins, G.S., Huang, L., Birch, L., and Pu, Y. 2006 The role of estrogens in normal and abnormal development of the prostate gland. Ann. N. Y. Acad. Sci. 1089:1-13.

22. Pu, Y., Huang, L., Birch, L., and Prins, G.S. 2007.
Androgen regulation of prostate morphoregulatory gene expression: Fof10-dependent and -independent pathways. Endocrinology. 148:1697-1706.

23. Krishnan, V., et al. 1997. Mediation of Sonic hedgehog-induced expression of COUP-TFII by a protein phosphatase. Science. 278:1947-1950.

24. Kim, B.J., Takamoto, N., Yan, J., Tsai, S.Y., and Tsai, M.J. 2008. Chicken ovalbumin upstream promotertranscription factor II (COUP-TFII) regulates growth and patterning of the postnatal mouse cerebellum. Dev. Biol. Online publication ahead of print. doi:10.1016/j.ydbio.2008.11.001.

25. Agha, A., and Monson, J.P. 2007. Modulation of glucocorticoid metabolism by the growth hormone - IGF-1 axis. Clin. Endocrinol. (Oxf). 66:459-465.

26. Hagg, T. 2007. Endogenous regulators of adult CNS neurogenesis. Curr. Pharm. Des. 13:1829-1840.

27. De Martino, M.U., Alesci, S., Chrousos, G.P., and Kino, T. 2004. Interaction of the glucocorticoid receptor and the chicken ovalbumin upstream promoter-transcription factor II (COUP-TFII): implications for the actions of glucocorticoids on glucose, lipoprotein, and xenobiotic metabolism. Ann. N. Y. Acad. Sci. 1024:72-85.

\title{
DAMPs ramp up drug toxicity
}

\author{
Jacquelyn J. Maher
}

Department of Medicine and Liver Center, UCSF, San Francisco, California, USA.

\begin{abstract}
The clinical syndrome of acetaminophen-induced liver injury represents the combined result of drug toxicity and a potent innate immune response that follows drug-induced cell death. In this issue of the JCI, Imaeda and colleagues report that DNA released from dying hepatocytes is a key stimulus of innate immune activation in the acetaminophen-treated mouse liver (see the related article beginning on page 305 ). They present evidence indicating that hepatocyte DNA promotes immune activation by acting as a danger-associated molecular pattern (DAMP) that stimulates cytokine production in neighboring sinusoidal endothelial cells via Tlr9 and the Nalp3 inflammasome.
\end{abstract}

The analgesic acetaminophen is widely known for its potential to cause severe and sometimes lethal liver injury. When ingested in large amounts, acetaminophen overwhelms the normal metabolic pathways of glucuronidation and sulfation and undergoes oxidation to form the highly reactive intermediate $N$-acetyl-p-benzoquinone-imine (NAPQI). NAPQI is not harmful if it combines rapidly with glutathione; however, when hepatic glutathione stores

Conflict of interest: The author has declared that no conflict of interest exists.

Nonstandard abbreviations used: DAMP, dangerassociated molecular pattern; HMGB1, high-mobility group box 1; NALP3, NACHT, LRR, and pyrin domain-containing protein 3; NAPQI, $N$-acetyl- $p$-benzoquinone-imine; NLR, nucleotide-binding domain, leucine-rich repeat-containing protein; SEC, sinusoidal endothelial cell.

Citation for this article: J. Clin. Invest. 119:246-249 (2009). doi:10.1172/JCI38178. are depleted, NAPQI escapes detoxification, resulting in liver cell death (1). An important but underappreciated aspect of acetaminophen toxicity is that direct, drug-induced harm accounts for only part of the overall syndrome of acetaminophen-induced liver injury. The reason for this is that the initial wave of drug-induced hepatocellular destruction is followed by a robust innate immune response, in which invading inflammatory cells release toxic oxidants and cause a second wave of destruction. The collateral damage inflicted by inflammatory cells can be so severe as to double the degree of tissue injury caused by acetaminophen alone (2).

\section{Innate immunity is the result of danger signaling}

Activation of the innate immune system following a noninfectious insult such as drug toxicity arises when dying cells release molecules that serve as "danger signals." Danger molecules trigger inflammation by engaging pattern recognition receptors such as TLRs (3) and nucleotide-binding domain, leucine-rich repeat-containing proteins (NLRs) (4) and are thus referred to as danger-associated molecular patterns (DAMPs) (5). Through TLRs, DAMPs signal cytokine and chemokine production and upregulate the expression of celladhesion molecules. When DAMPs interact with NLRs, they stimulate NLRs to complex with ASC (apoptosis-associated speck-like protein containing a CARD) to form macromolecular complexes called inflammasomes, which activate caspase-1 and stimulate cleavage of the proinflammatory cytokines pro-IL- $1 \beta$ and pro-IL-18 to their mature forms, IL-1 $\beta$ and IL-18 (6). Self molecules that act as DAMPs interact primarily with TLR2, -4 , and -9 and an NLR with an $\mathrm{N}$-terminal pyrin domain designated NACHT, LRR, and pyrin domain-containing protein 3 (NALP3; also known as NLRP3). The list of these molecules is rapidly growing (Table 1), emphasizing the importance of endogenous danger signaling to a broad array of medical disorders ranging from gout to systemic lupus erythematosus to Alzheimer disease (7-9). A danger molecule that is believed to play a 
Table 1

Self molecules that interact with TLRs and NLRs

\begin{tabular}{llc}
\hline & & \\
SLlf molecule & Activates \\
& HMGB1 & TLR2, $-4,-9$ \\
& DNA & TLR9 \\
& Heat shock proteins & TLR2, -4 \\
& Defensins & TLR4 \\
& ATP & NALP3 \\
& Monosodium urate crystals & NALP3 \\
& Calcium pyrophosphate dihydrate crystals & NALP3 \\
& Silica crystals & NALP3 \\
& Alum particles & NALP3 \\
& Amyloid- $\beta$ particles & NALP3 \\
& Asbestos particles & NALP3 \\
\hline
\end{tabular}

central role in inflammatory diseases of the liver is the chromatin-binding protein high-mobility group box 1 (HMGB1). Upon cellular necrosis, HMGB1 is released into the extracellular milieu, where it activates TLR2 and TLR4 and, when complexed with DNA, TLR9 $(10,11)$. Studies to date indicate that HMGB1 plays an important role in liver injury due to hemorrhagic shock and ischemia/reperfusion (12), but, interestingly, it does not strongly influence acetaminophen toxicity (13).

\section{Figure 1}

DNA-mediated danger signaling in acetaminophen toxicity. Acetaminophen is directly cytotoxic to hepatocytes through its conversion to the reactive intermediate NAPQI (1). In this issue of the $\mathrm{JCl}$, Imaeda and colleagues provide evidence that DNA released from acetaminophen-damaged hepatocytes induces an innate immune response in the liver that augments the injury caused by the drug alone (18). They show in a mouse model that acetaminophen, which damages hepatocytes, as well as damaged DNA per se, activates TIr9 within SECs, thereby stimulating the production of pro-IL-1 $\beta$ and pro-IL-18. Acetaminophen treatment also promotes activation of the Nalp3 inflammasome in SECs, whose function is to activate caspase- 1 and promote the cleavage of pro-IL-1 $\beta$ and pro-IL-18 to their mature, active forms (6). (The specific stimulus to Nalp3 activation in the acetaminophen-treated liver is unknown, but potential candidates include uric acid, ATP, and DNA.) $\mathrm{IL}-1 \beta$ and IL-18 in turn enhance acetaminophen-induced liver injury by promoting hepatic inflammation and secondary tissue damage. Imaeda et al. further demonstrate that this immune-mediated amplification of acetaminophen-induced liver injury can be blocked by aspirin. Aspirin prevents hepatic induction of pro-IL-1 $\beta$ and pro-IL-18 and may have an independent inhibitory effect on the Nalp3 inflammasome.

\section{Danger signaling in acetaminophen hepatotoxicity}

Although the precise nature of the danger signal that activates innate immunity in the acetaminophen-exposed liver is uncertain, there is no question that danger signaling is involved in the process. This is evident from studies in a mouse strain with a mutation in Tlr4, in which liver disease is significantly attenuated following an acetaminophen challenge (14). Recently, acetaminophen toxicity was shown to be reduced in mice that lack the ability to respond to IL-1 (15). This observation is of interest because IL-1 secretion typically requires the combined activity of TLRs and NLRs, the former to stimulate expression of the IL-1 propeptide and the latter to process the propeptide into a mature cytokine. It also suggests a need for two, possibly unique, danger signals in the liver: one to activate a TLR and another to signal NLR assembly into an inflammasome (6).

If the danger signal that augments acetaminophen-induced liver injury is not HMGB1, then another molecule that could accomplish this task is DNA from dying hepatocytes. DNA interacts specifically with TLR9, which, like all nucleic acidsensing TLRs, is sequestered intracellularly within endosomes. TLR9 was once con-

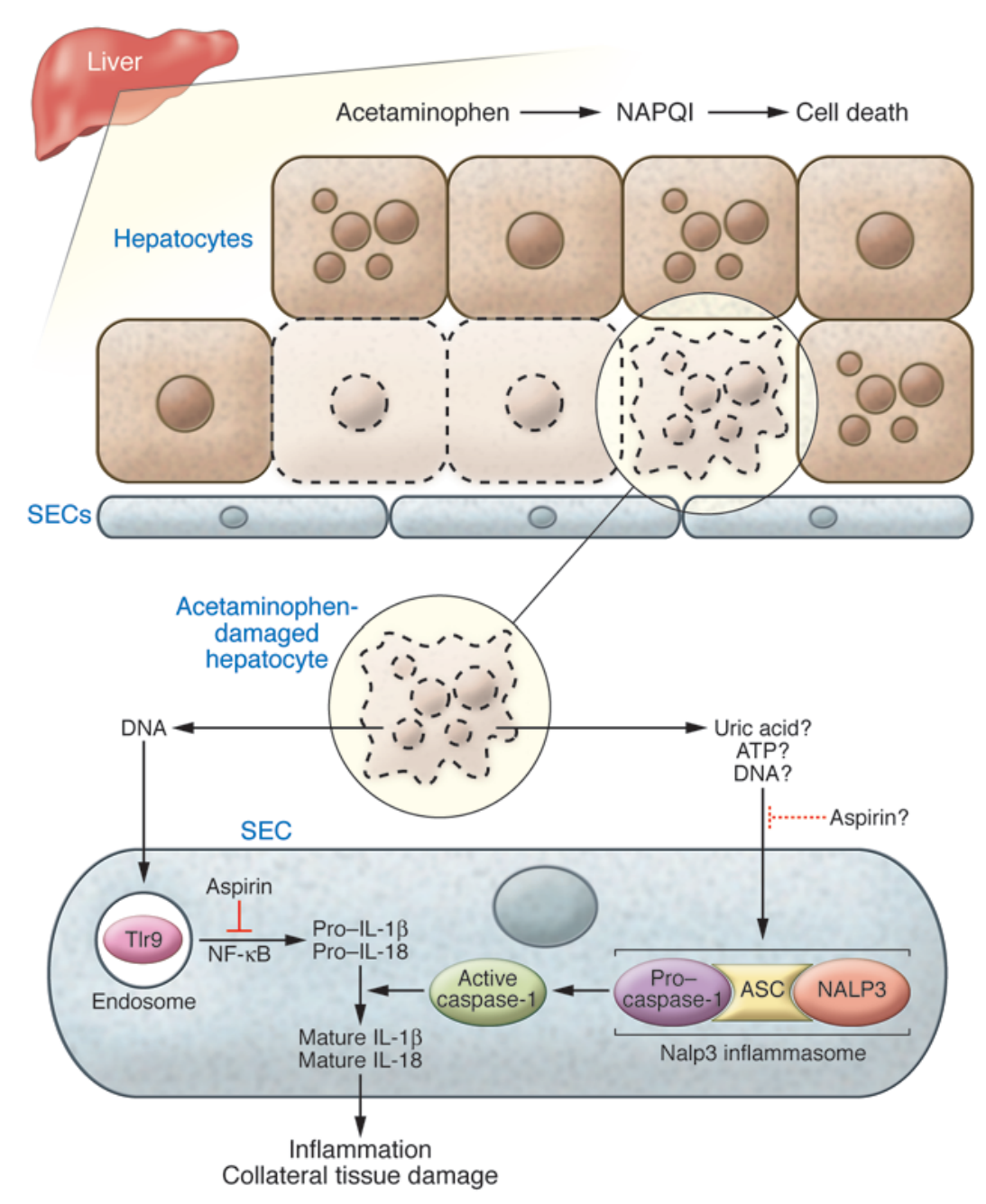


sidered incapable of binding mammalian DNA because of its affinity for unmethylated CPG motifs characteristic of microbial DNA. DNA from injured mammalian cells, however, has the capacity to activate TLR9 $(8,16)$, and recently even normal mammalian DNA has been shown to engage this receptor and stimulate an immune response (17). In this issue of the JCI, Imaeda and colleagues (18) demonstrate that DNA is indeed a trigger of the innate immune response that amplifies acetaminophen toxicity. They showed, in a mouse model, that acetaminophen-induced liver injury is dependent upon not only IL- $1 \beta$, but also IL-18, the two cytokines classically processed by caspase-1 (Figure 1). They determined that acetaminophen-mediated induction of pro-IL-1 $\beta$ and pro-IL-18 mRNA in the liver is Tlr9 dependent; in addition, they showed that cleavage of the IL- $1 \beta$ propeptide to the mature cytokine in the acetaminophentreated liver requires the presence of the Nalp3 inflammasome. Inhibition of either Tlr9 signaling or Nalp3 activity by genetic or pharmacologic means markedly attenuated liver injury and improved survival in acetaminophen-treated mice. These results indicate that Tlr9 and Nalp 3 must both be functional to activate the innate immune system following acetaminophen exposure, and they underscore the degree to which immune-mediated collateral damage intensifies drug-induced liver injury.

Imaeda et al. (18) did not demonstrate that DNA released specifically from dying hepatocytes is the stimulus for Tlr9 activation in the liver following acetaminophen treatment. They did, however, demonstrate that DNA from dead (UV-irradiated) hepatocytes upregulates IL- $1 \beta$ in a Tlr9dependent manner when infused into a normal liver in vivo. They also made the unique observation that much of the innate immune response to acetaminophen poisoning is mediated by sinusoidal endothelial cells (SECs), which are actively endocytic cells. TLR9 is expressed by several resident liver cells, including hepatocytes, SECs, Kupffer cells, and stellate cells, but among these, SECs exhibit the greatest potential for taking up extracellular DNA (19). Purified SECs displayed DNA-mediated activation of Tlr9 and acetaminophen-mediated activation of caspase-1 (18), which pinpoints these cells as immune effectors in acetaminophen toxicity and places them in a category with bone marrow-derived cells as sites of the molecular machinery of the inflammasome.
Viewing immune amplification of acetaminophen-induced liver injury as a therapeutic target, Imaeda and colleagues subsequently investigated whether the immune-mediated collateral damage in an acetaminophen-treated liver was preventable by administration of an antiinflammatory agent (18). Aspirin, when given before acetaminophen, significantly protected mice against acetaminophen-induced liver injury. Indeed, aspirin-pretreated mice fared as well or better after acetaminophen challenge than mice lacking Tlr9 or components of the Nalp3 inflammasome. The protective effect of aspirin coincided with reduced induction of hepatic pro-IL- $1 \beta$ and pro-IL-18 mRNA after acetaminophen administration. This suggests that aspirin is exerting its protective effect on the Tlr9 arm of the danger pathway during acetaminophen toxicity, perhaps by inhibiting IкB kinase $\beta$ (20). Aspirin also inhibited experimental inflammation induced by monosodium urate crystals, which activate Nalp3, but it remains unclear whether this is interpretable as an independent effect of aspirin on caspase- 1 activation by the Nalp3 inflammasome (Figure 1).

\section{Novel concepts and open questions}

Overall, the work of Imaeda and colleagues (18) sheds important light on the role of the inflammasome in drug-induced liver injury. It highlights potential differences between innate immune responses to different stimuli (e.g., drugs versus ischemia/reperfusion) and places SECs in a category with bone marrow-derived cells as orchestrators of innate immune responses to DNA. Still, there remain some unanswered questions. One is whether bone marrow-derived cells, which also contribute to innate immune activation in response to acetaminophen, sense DNA as their danger signal in the same fashion as SECs. Another is whether DNA from hepatocytes treated with acetaminophen has any unique characteristics with respect to Tlr9 activation. Third, one wonders whether immune-mediated collateral damage in acetaminophen toxicity is due entirely to leukocyte invasion, or whether inflammasome-mediated cell death is also involved. Under certain circumstances, NALP3, ASC, and caspase-1 can interact to cause cell death (21). Such a pathway could be operative in SECs during acetaminophen toxicity. If so, this may explain why innate immune activation in the liver is so often accompanied by sinusoidal cell breakdown and parenchymal hemorrhage, which in many animal models of acute liver injury is the fatal event.

Imaeda and coworkers (18) posited that two separate danger signals are required to activate IL-1 $\beta$ and IL-18 in the acetaminophen-treated liver: hepatocyte DNA to induce cytokine gene expression via Tlr9 and another molecule, possibly uric acid or ATP, to activate the Nalp3 inflammasome and promote cytokine cleavage. This theory was logical, based on evidence that few if any compounds stimulate both TLRs and NLRs. Recent work by Muruve et al. (22), however, indicates that mammalian DNA can promote inflammasome formation, albeit in the absence of NALP3. Further research in this area may ultimately lead to a unified theory according to which self DNA activates not only TLR9 but also the NALP3 inflammasome following a cytotoxic insult in vivo, culminating in immune-mediated collateral damage to the affected organ.

\section{Acknowledgments}

This work is supported by grants R01 DK061510, R01 DK068450, and P30 DK026743 (UCSF Liver Center).

Address correspondence to: Jacquelyn J. Maher, Rice Liver Center Laboratory, San Francisco General Hospital, 1001 Potrero Avenue, Building 40, Room 4102, San Francisco, California 94110, USA. Phone: (415) 206-4805; Fax: (415) 641-0517; E-mail: jmaher@medsfgh.ucsf.edu.

1. Gunawan, B.K., and Kaplowitz, N. 2007. Mechanisms of drug-induced liver disease. Clin. Liver Dis. 11:459-475.

2. Liu, Z.X., Han, D., Gunawan, B., and Kaplowitz, N. 2006. Neutrophil depletion protects against murine acetaminophen hepatotoxicity. Hepatology. 43:1220-1230.

3. Kawai, T., and Akira, S. 2006. TLR signaling. Cell Death Differ. 13:816-825

4. Ye, Z., and Ting, J.P. 2008. NLR, the nucleotidebinding domain leucine-rich repeat containing gene family. Curr. Opin. Immunol. 20:3-9.

5. Bianchi, M.E. 2007. DAMPs, PAMPs and alarmins: all we need to know about danger. J. Leukoc. Biol. 81:1-5.

6. Mariathasan, S., and Monack, D.M. 2007. Inflammasome adaptors and sensors: intracellular regulators of infection and inflammation. Nat. Rev. Immunol. 7:31-40.

7. Halle, A., et al. 2008. The NALP3 inflammasome is involved in the innate immune response to amyloid-beta. Nat. Immunol. 9:857-865.

8. Lamphier, M.S., Sirois, C.M., Verma, A., Golenbock, D.T., and Latz, E. 2006. TLR9 and the recognition of self and non-self nucleic acids. Ann. N. Y. Acad. Sci. 1082:31-43.

9. Martinon, F., Petrilli, V., Mayor, A., Tardivel, A., and Tschopp, J. 2006. Gout-associated uric acid crystals activate the NALP3 inflammasome. Nature. 440:237-241. 
10. Park, J.S., et al. 2004. Involvement of toll-like receptors 2 and 4 in cellular activation by high mobility group box 1 protein. J. Biol. Chem. 279:7370-7377.

11. Tian, J., et al. 2007. Toll-like receptor 9-dependent activation by DNA-containing immune complexes is mediated by HMGB1 and RAGE. Nat. Immunol. 8:487-496.

12. Tsung, A., et al. 2005. The nuclear factor HMGB1 mediates hepatic injury after murine liver ischemiareperfusion. J. Exp. Med. 201:1135-1143.

13. Scaffidi, P., Misteli, T., and Bianchi, M.E. 2002. Release of chromatin protein HMGB1 by necrotic cells triggers inflammation. Nature. 418:191-195.

14. Yohe, H.C., et al. 2006. Involvement of Toll-like receptor 4 in acetaminophen hepatotoxicity. Am.J. Physiol. Gastrointest. Liver Physiol. 290:G1269-G1279.

15. Chen, C.J., et al. 2007. Identification of a key pathway required for the sterile inflammatory response triggered by dying cells. Nat. Med. 13:851-856.

16. Ishii, K.J., et al. 2001. Genomic DNA released by dying cells induces the maturation of APCs. J. Immunol. 167:2602-2607.

17. Haas, T., et al. 2008. The DNA sugar backbone 2 ' deoxyribose determines toll-like receptor 9 activation. Immunity. 28:315-323.

18. Imaeda, A.B., et al. 2009. Acetaminophen-induced hepatotoxicity in mice is dependent on $\operatorname{Tl} 9$ and the Nalp3 inflammasome. J. Clin. Invest. 119:305-314.
19. Martin-Armas, M., et al. 2006. Toll-like receptor 9 (TLR9) is present in murine liver sinusoidal endothelial cells (LSECs) and mediates the effect of CpG-oligonucleotides. J. Hepatol. 44:939-946.

20. Yin, M.J., Yamamoto, Y., and Gaynor, R.B. 1998. The anti-inflammatory agents aspirin and salicylate inhibit the activity of I(kappa)B kinase-beta. Nature. 396:77-80.

21. Ting, J.P., Willingham, S.B., and Bergstralh, D.T. 2008. NLRs at the intersection of cell death and immunity. Nat. Rev. Immunol. 8:372-379.

22. Muruve, D.A., et al. 2008. The inflammasome recognizes cytosolic microbial and host DNA and triggers an innate immune response. Nature. 452:103-107.

\title{
Hoofbeats, zebras, and insights into insulin resistance
}

\author{
Robert A. Hegele ${ }^{1}$ and Karen Reue ${ }^{2}$ \\ ${ }^{1}$ Robarts Research Institute, University of Western Ontario, London, Ontario, Canada. ${ }^{2}$ Department of Human Genetics, \\ UCLA David Geffen School of Medicine, Los Angeles, California, USA.
}

\begin{abstract}
In this issue of the JCI, Semple and colleagues report phenotypic evaluation of patients with a germline mutation in the gene encoding serine/threonine kinase AKT2 (see the related article beginning on page 315). Their findings support the idea that the postreceptor actions of insulin in the liver - suppression of gluconeogenesis and stimulation of lipogenesis - are mediated through divergent pathways that can be uncoupled. The results appear to refine the arrangement of crucial steps along these pathways and show how comprehensive study of the phenotype, "deep phenotyping," of patients who carry rare mutations might complement other types of experiments to elucidate complex pathways and mechanisms.
\end{abstract}

"When you hear hoofbeats, think horses, not zebras" is the quintessential maxim of clinical medicine. But in clinical investigation, the "zebras" - rare conditions that recapitulate, often to an extreme, the components of a common disease - can help to understand the "horse" or common complex phenotype. Extending the metaphor, the current pandemic of obesity and insulin resistance is a veritable stampede that threatens to flatten global medical care infrastructures. A multifaceted approach is required to understand the mechanisms underlying this pandemic, ranging from the strategic use of model systems to population studies and clinical trials. Within this methodological spectrum is the evolving discipline of clinical phenomics, which

Conflict of interest: The authors have declared that no conflict of interest exists.

Nonstandard abbreviations used: FOXO1, forkhead box $\mathrm{O}$ transcription factor 1 ; INSR, insulin receptor; TG, triglyceride.

Citation for this article: J. Clin. Invest. 119:249-251 (2009). doi:10.1172/JCI38420. uses objective and systematic acquisition of phenotypic data (i.e., deep phenotyping) of selected informative patients (1). Phenomic evaluation of patients with rare genetic disorders is a potential tool to help solve the puzzle of insulin resistance and its downstream metabolic consequences, such as hyperglycemia, hepatosteatosis and dyslipidemia, elevated triglyceride (TG) levels, and depressed HDL cholesterol levels.

\section{Insulin-resistant diabetes: a disease of abnormal glucose and lipid metabolism}

The complex web of interactions between glucose and lipid metabolism in diabetes has long been appreciated, if incompletely understood mechanistically (2-5). Inducedmutant animal models have steadily advanced our understanding of insulin resistance. For instance, insulin-resistant mice with a liver-specific deletion of the insulin receptor (INSR) develop hyperglycemia but not dyslipidemia (6). This suggests an uncoupling or divergence (see Fig- ure 1) between the post-INSR pathways, with loss of insulin-mediated suppression of gluconeogenesis normally driven by phosphoenolpyruvate decarboxylase and glucose-6-phosphatase, but retention of insulin's stimulatory effect on hepatic lipogenesis catalyzed by fatty acid synthases (7). Signaling intermediates along the gluconeogenesis limb include serine/threonine kinase AKT2 (also called phosphokinase B) and forkhead box $\mathrm{O}$ transcription factor 1 (FOXO1), while intermediates along the de novo hepatic lipogenesis limb include PKC $\lambda$ and SREBP-1c $(8,9)$. To clarify the basis of such "asymmetric" or partial insulin resistance, deep phenotyping of patients carrying naturally occurring loss-of-function mutations in these intermediate signaling molecules might be instructive.

In their current study in this issue of the JCI, Semple and colleagues (10) have attempted to probe these pathways by studying patients with extremely rare mutations in either INSR or AKT2 genes together with subjects who had either idiopathic insulin resistance or inherited lipodystrophies. The study took advantage of a valuable archive of phenotypically and molecularly characterized patients who were carefully collected over many years. Among patients with INSR mutations and anti-INSR antibodies, Semple and colleagues first confirmed earlier work that showed severe hyperglycemia and hyperinsulinemia but normal plasma lipids in these patients (11). These findings are 